\title{
Publisher's Note: Critical behavior of the quasi-two-dimensional weak itinerant ferromagnet trigonal chromium telluride $\mathrm{Cr}_{0.62}$ Te [Phys. Rev. B 96, 134410 (2017)]
}

\author{
Yu Liu (刘育) and C. Petrovic
}

Q (Received 10 January 2020; published 16 January 2020)

DOI: 10.1103/PhysRevB.101.049904

This paper was published online on 9 October 2017 and subsequently corrected on 29 November 2018. Some statements in the Acknowledgment section require further updating. The Acknowledgments should read as "This work was supported by the US Department of Energy, Office of Basic Energy Sciences as part of the Computational Material Science Program.” The paper has been corrected as of 14 January 2020. The text is incorrect in the printed version of the journal. 